\title{
Preliminary Findings on Visual Event-Related Potential P3 in Asymptomatic Patients with Cerebral Small Vessel Disease
}

\author{
Shanjing $\mathrm{Nie}^{\mathrm{l-3}}$ \\ Chao Shen ${ }^{1,2}$ \\ Yunliang Guo ${ }^{1-3}$ \\ Xunyao Hou ${ }^{\mathrm{I} 3}$ \\ Yan Hong ${ }^{1-3}$ \\ Song $\mathrm{Xu}^{\mathrm{I}-3}$ \\ Renjun $\mathrm{Lv}^{4}$ \\ Xueping Liu ${ }^{1-3}$
}

'Department of Geriatrics, Shandong Provincial Hospital Affiliated to Shandong First Medical University, Jinan, Shandong, People's Republic of China; ${ }^{2}$ Department of Geriatric Neurology, Shandong

Provincial Hospital Affiliated to Shandong First Medical University, Jinan, Shandong, People's Republic of China; ${ }^{3}$ Anti-Aging Monitoring Laboratory, Shandong Provincial Hospital Affiliated to Shandong First Medical University, Jinan, Shandong, People's Republic of China; ${ }^{4}$ Shandong Provincial Hospital, Shandong First Medical University \& Shandong Academy of Medical Sciences, Jinan, Shandong, People's Republic of China
Correspondence: Xueping Liu Department of Senile Neurology, Shandong Provincial Hospital Affiliated to Shandong First Medical University, Jinan, 25002I, Shandong, People's Republic of China

Email Liuxueping1962@163.com
Background: Cerebral small vessel disease is the primary cause of cognitive impairment. Therefore, early recognition is of great significance. Some studies have shown that asymptomatic cerebral small vessel disease (aCSVD) patients have abnormal neurocognitive function, but this is not readily apparent at the initial stage. The objective of this paper was to assess visual spatial attention by event-related potential (ERP) examination and to analyze the relationship between ERP data and clinical characteristics in patients with aCSVD.

Methods: We selected 25 aCSVD patients and enrolled 23 age-matched normal subjects as the control group. We measured the latency and amplitude of original/corresponding differential ERP components using the modified visual oddball paradigm, which included a standard stimulus, target stimulus, and new stimulus. Additionally, we selected aberrant ERP components to study the correlations between the ERP data and clinical characteristics of the patients with aCSVD.

Results: We found not only lower amplitude but also significantly longer P3 latency in the aCSVD patients. The above results were further verified by analyzing the different components (target minus standard and novel minus standard) of P3. Furthermore, abnormal ERPs in the aCSVD patients were closely related to the changes observed with imaging.

Conclusion: It was demonstrated that the speed and capability of processing visual spatial information was impaired in aCSVD patients compared with healthy controls. Thus, ERP examination could detect the presence of attentional deficits and might become a rapid and sensitive method for the early diagnosis of aCSVD. However, its availability needs further investigation.

Keywords: asymptomatic cerebral small vessel, event-related potential, cognitive impairment, P3, visual spatial attention impairment

\section{Introduction}

Cerebral small vessel disease is one of the main causes of ischemic stroke ${ }^{1,2}$ and is a hotspot in current research. The pathology of cerebral small vessel disease is predominantly characterized by endothelial dysfunction, blood brain barrier breakdown and small vessel wall degeneration. Generally, pathological changes not only lead to brain parenchymal injury but also cause neurological symptoms, as well as changes in neuroimaging. ${ }^{3}$ The ischemic mechanism caused by endothelial dysfunction has been confirmed in experimental animal models; for instance, overexpression of the p53 gene can mediate apoptosis of oligodendrocytes, leading to demyelination. ${ }^{4}$ Research has shown that in rats, the permeability of the blood-brain barrier is disturbed. ${ }^{5}$ Recent 
studies in mice with single penetrating arteriole occlusion have found that local microvascular dysfunction leads to tissue damage. ${ }^{6}$ Although the pathogenesis of cerebral small vessel disease is diverse, neuroimaging markers are similar, including recent small subcortical infarcts, lacunes, white matter hyperintensities, perivascular spaces, microbleeds, and brain atrophy. ${ }^{7}$ The clinical manifestations of cerebral small vessel disease lack specificity and mainly include stroke symptoms, cognitive and emotional symptoms, and general dysfunction (urination and defecation symptoms, gait disorders), while some patients have only imaging markers. ${ }^{8}$

Remarkably, cerebral small vessel disease is the primary cause of vascular cognitive impairment, ${ }^{9}$ which includes all stages of cognitive function, from normal to complete dementia. ${ }^{10}$ In most cases, cerebral small vessel disease is only recognized in its advanced stages once its symptomatic sequelae develop; studies related to cognition have also focused on this topic of interest, which is characterized by memory decline. ${ }^{11}$

Asymptomatic cerebral small vessel disease (aCSVD) is defined as neuroimaging evidence of cerebral small vessel disease prior to the development of any overt clinical symptoms. However, aCSVD is not without any impact on people. There is emerging evidence that brain structure $^{12}$ and corresponding function ${ }^{13}$ have already changed by this stage. The associated cognitive decline predominantly involves problems with executive function and with attention and information processing speed, ${ }^{14}$ which are basic cognitive processes of the brain. ${ }^{15}$ Consequently, such changes in normal cognitive performance tend to be regarded as a function of the normal aging process and are often ignored by patients and their families and medical workers. Here, we will investigate aCSVD, with a focus on its early prevention and on reducing its burden on patients' families and society.

One of the greatest challenges is that there are limited means to assess aCSVD. Currently, the diagnosis and classification of cognitive impairment are still mainly based on clinical manifestations, neuropsychological scales, subjective reports by family members on the individual's cognitive decline, and imaging indicators, which are all ambiguous and delayed while restricting early clinical diagnosis and prevention. Nevertheless, an eventrelated potential (ERP) examination, with the associated characteristics of objectivity and high temporal resolution, offers great advantages in revealing the temporal aspects of cognition and is widely used for detecting early cognitive impairment. ERP is likely to detect subtle changes in cognitive function in those who perform normally on standardized scales. ${ }^{16}$ Evidence-based research confirms that P3 is the most studied component and is a sensitive measurement index for early changes in cognitive function in the elderly.

In this study, our aim was to observe the characteristics of ERP data induced by the improved visual oddball paradigm in aCSVD patients, distinguish those with aCSVD from healthy counterparts, and investigate the correlations between ERP data and the clinical features of aCSVD patients. We hypothesized that aCSVD patients have deficits in attentional processing and abnormal changes in corresponding ERP components.

\section{Methods}

\section{Subjects and Criteria}

Thirty-two aCSVD patients (18 males, 14 females) were recruited for the present study from Shandong Provincial Hospital Affiliated to Shandong First Medical University. In addition, 27 age-matched healthy controls (12 males, 15 females) from this hospital staff or volunteers from the community were enrolled in the research.

For aCSVD patients, the inclusion criteria were as follows: (1) the patient's brain changes on magnetic resonance imaging corresponded to cerebral small vessel disease (Wardlaw et al, 2013), (2) the definitions of lacuna and white matter hyperintensity (WMH) were in accordance with the reporting criteria of the vascular changes proposed by Wardlaw et al, ${ }^{17}$ and (3) the Fazekas scale was used to quantify T2-weighted FLAIR WMH. ${ }^{18}$ The exclusion criteria were (1) intracranial hemorrhage, (2) coronary atherosclerotic heart disease or carotid stenosis ( $>75 \%)$, (3) other nervous system diseases, such as Parkinson's disease, Alzheimer's disease, and epilepsy, (4) loss of vision or hearing, and (5) pseudocognitive impairment caused by depression and anxiety.

Within the study, all scales were evaluated by trained physicians using standardized questionnaires, and their clinical characteristics were obtained, including scores relating to the Mini-Mental State Examination (MMSE), Montreal Cognitive Assessment (MoCA), Patient Health Questionnaire-9 (PHQ-9), Generalized Anxiety Disorder-7 (GAD-7), and Fazekas scale. The cognitive screening tool, using MMSE and MoCA. Additionally, the PHQ-9 and GAD-7 were used for the quantitative evaluation of 
emotion, and the Fazekas scale was employed to quantify alterations in brain magnetic resonance imaging.

Furthermore, we excluded seven aCSVD patients (4 females, 3 males). Of these, four patients had excessive blink and myoelectric activity in their electroencephalogram data, two had incomplete clinical data and one patient was excluded because of data problems. Thus, 25 subjects (10 females, 15 males) with aCSVD were enrolled in the study. For the controls, four participants ( 2 females, 2 males) were excluded, one for a lack of image data, the other two for technical problems in the recording process and one on account of the data. Finally, we selected 23 participants (13 females, 10 males) as the control group.

The research program was approved by the Ethical Committee of Shandong Provincial Hospital Affiliated to Shandong First Medical University (SWYX: NO.2020 232), which was in line with the ethical principles of the Helsinki declaration. Before testing, all subjects signed an informed consent form.

\section{Neuropsychological Assessments and Emotional Evaluations}

The MMSE scale is regarded as the most commonly used standardized cognitive assessment test. ${ }^{19}$ The MoCA evaluation is a multidomain cognitive screening instrument as well, with high sensitivity to suspected mild cognitive decline. ${ }^{20}$ In our study, these two examinations were conducted on the same day with an interval of more than 3 hours, and the MMSE and MoCA scores were within the normal range.

Emotional state was assessed using GAD-7 and PHQ-9 scales. The GAD-7 is a self-rating scale comprising seven items and is a reliable tool for diagnosing generalized anxiety disorders. ${ }^{21}$ The PHQ-9 comprises nine items and can be used to evaluate depressive disorder. ${ }^{22}$ All participants were required to answer each item of the aforementioned two scales for nearly 2 weeks, and their answers were rated on a 4-point scale, ranging from 0 (not at all) to 3 (nearly every day). The total scores were summed and calculated, with higher scores indicating increased severities of anxious and depressive symptoms. The critical scores were set at 4 to test the anxiety and depression levels of aCSVD patients.

\section{Imaging Feature Evaluation}

The Fazekas scale is a visual semiquantitative method for assessing WMH and has been proven to have higher efficacy than other scales. ${ }^{23}$ It is widely employed in evaluating cerebral small vessel disease patients, ${ }^{24,25}$ as it correlates well with the extent of lesions determined by pathological analysis. ${ }^{26}$ In our study, WMHs were evaluated on T2 FLAIR images using the Fazekas scale (Grade 0 , absent or dot-shaped signal; Grade 1, multiple dotshaped lesions; Grade 2, unified lesions; Grade 3, large confluent or severe lesions). ${ }^{27}$ The assessments were performed by two experienced senior experts who were familiar with cerebral small vessel disease (XL and $\mathrm{XH})$.

\section{Electrode Placement and Electroencephalogram Recording}

The experimental room was kept dark and quiet, with no electronic equipment, which can cause interference. All subjects were relaxed in a chair and were asked to focus their eyes on a fixed cross $50 \mathrm{~cm}$ (23 inches) from the center of the screen. Before the test, they were given detailed instructions by the experimenters and were required to blink less, relax their muscles, and reduce their swallowing.

Electroencephalograms of all subjects were recorded with a Neurolab EEG/ERPs 32 Channel Amplifier system using an $\mathrm{Ag}-\mathrm{AgCl}$ active electrode, according to the international 10-20 system. Recordings were obtained from 32 electrode sites, and we regarded the left mastoid signal as the reference electrode. We wiped the corresponding electrode position on the scalp with alcohol to exfoliate it and ensure that the electrode impedance remained below 10 $\mathrm{k} \Omega$ throughout the entire process. Eye movement was recorded by electrodes at the right orbital $10 \mathrm{~mm}$ from the lateral canthus. The sampling rate of the whole experiment was set at $1000 \mathrm{~Hz}$, and the low-pass filter was set at $100 \mathrm{~Hz}$. Finally, according to the previous literature, ${ }^{28}$ we conducted statistical analyses on the data from the $\mathrm{Fz}, \mathrm{Cz}$, and Pz.

\section{ERP Task Stimulus Sequence}

Our experiment, which was based on the modified visual oddball paradigm, was divided into three parts. A total of 501 stimuli were randomly presented, including a standard stimulus (small circle, $\mathrm{n}=381$ ), target stimulus (large circle, $\mathrm{n}=60$ ), and novel stimulus (chessboard, $\mathrm{n}=60$ ). The duration of stimulation was $400 \mathrm{~ms}$, and the interval between each stimulus was $400 \mathrm{~ms}$. The target stimulus appeared 60 times in total, 20 times per module. Participants were asked to silently count the number of 
large circles (target stimuli) in each part and report this figure to the doctor after the experiment.

\section{ERP Data Handling}

ASA 4.9.3 software was used to analyze the electroencephalogram data offline. The data were compared with the average value of a bilateral mastoid signal. Independent component analysis, as proposed by Jung et al, ${ }^{29}$ was used to detect electrooculogram activity, and the bandpass filter was set at $0.1-30 \mathrm{~Hz}(24 \mathrm{~dB} /$ octave). Extracting epochs from $200 \mathrm{~ms}$ before stimulation to $1000 \mathrm{~ms}$ post stimulation, we used a baseline of -200 to $0 \mathrm{~ms}$ to correct and measure all ERP waveforms. In any recording channel, any test with a signal amplitude exceeding $\pm 100 \mu \mathrm{V}$ was rejected for averaging. Finally, the waveform of a single subject generated the total average waveform, which was then further analyzed.

Based on the baseline period before stimulus, we measured the peak amplitude and latency of ERPs. ERP components in this study included the following: P3-a positive peak produced approximately $300-500 \mathrm{~ms}$ after stimulation; N1-a negative peak appearing 50-190 ms after stimulation; $\mathrm{P} 2$ - a positive peak provoked approximately 110-270 ms after stimulation; and N2-a negative peak appearing 210-370 ms after stimulation. ${ }^{30}$

To observe the effects of target and novel stimuli more intuitively, we subtracted the response to the standard stimulus from the response to the target stimulus and the response to the novel stimulus, calculated and plotted the difference waveform of ERP, and further analyzed the difference ERP data.

The measurements and analyses were carried out blindly by inspectors.

\section{Statistical Analysis}

All statistical analyses were carried out using SPSS 23.0 software (SPSS Inc., Chicago, IL, USA). For comparison between the two groups, an independent samples $t$-test was used for quantitative data, chi square test (sex) was used for counting data, and nonparametric test was used for count data (Fazekas). In addition, the normality of the ERP data was tested, and then repeated-measures analysis of variance (ANOVA) was employed to analyze the amplitude and latency of the original ERP component (P3, N1, $\mathrm{P} 2$, N2), with stimuli (standard, target and novel) and electrodes $(\mathrm{Fz}, \mathrm{Cz}$ and $\mathrm{Pz})$ being within-subject factors, while the group (aCSVD patients vs healthy controls) was taken as a between-subject factor. More specifically, for the $\mathrm{P} 3$ component, $\mathrm{P} 3$ elicited by the target was defined as $\mathrm{P} 3 \mathrm{~b}$, and P3a components elicited by novel stimuli were defined. For differential components of ERP $\left(\mathrm{P}^{2} \mathrm{~d}_{\mathrm{T}}\right.$ : target minus standard; $\mathrm{P} 3 \mathrm{~d}_{\mathrm{N}}$ : novel minus standard), electrodes $(\mathrm{Fz}, \mathrm{Cz}$ and $\mathrm{Pz}$ ) were within-subject factors, while group (aCSVD patients and healthy control group) was a between-subject factor. The Greenhouse-Geisser epsilon was used to correct the degree of freedom in the case of violation of the spherical assumption. Bonferroni correction, if required, was used for further post hoc analysis. Pearson correlation analysis was used to analyze the correlation between ERP variables and quantitative data; Spearman rank correlation analysis was used to analyze the correlation between ERP variables and Fazekas. " $r$ " is the correlation coefficient. The $P$ value was less than 0.05 , with statistical significance. The effect size of significant results was reported as partial eta squared (partial $\eta^{2}$ ) for ANOVA.

\section{Results}

\section{Sample Characteristics}

The sample characteristics of the subjects are listed in Table 1. Among them, age, sex, and education years did not differ between the two groups (all $\mathrm{P}>0.05$ ). In terms of neuropsychological assessments and emotional characteristics, there was no statistically significant difference between the two groups $(\mathrm{P}>0.05)$.

\section{Characterization of Original ERP Data}

Figure 1 depicts the grand average waveforms of the original ERP, and Table 2, Figures 2, and 3 summarize several original ERP components.

\section{P3 Component}

Figure 4 shows topographic maps of the voltage distribution for P3 induced by standard, target (P3b), and novel stimuli (P3a). aCSVD patients showed a lower P3 amplitude (5.169 \pm $4.188 \mu \mathrm{V})$ than healthy controls $(7.909 \pm 5.767 \mu \mathrm{V}, \mathrm{F}(1,46)=$ $18.763, \mathrm{P}=0.000$, partial $\left.\eta^{2}=0.290\right)$. There was a significant main effect of stimulus $(\mathrm{F}(2,92)=103.652, \mathrm{P}<0.001$, partial $\left.\eta^{2}=0.693\right)$, and post hoc comparisons found that P3a elicited by novel stimuli was the largest $(9.455 \pm 4.418 \mu \mathrm{V})$. Moreover, a remarkable group $\times$ stimulus interaction $(\mathrm{F}(2,92)=4.422, \mathrm{P}$ $=0.015$, partial $\eta^{2}=0.088$ ) was displayed (Table 2). Furthermore, post hoc analysis showed that the P3a amplitude (elicited by novel stimuli) was lower in the aCSVD group ( $\mathrm{F}$ $(1,46)=13.521, P=0.001$, partial $\left.\eta^{2}=0.227\right)$. Compared with the control group, the amplitude of $\mathrm{P} 3 \mathrm{~b}$ (elicited by target 
Table I Sample Characteristics

\begin{tabular}{|l|l|l|l|l|}
\hline & Healthy Controls & aCSVD Patients & $\boldsymbol{t}$ l $\boldsymbol{2}$ 2 & $\boldsymbol{P}$ \\
\hline Number & 23 & 25 & & 0.944 \\
Age, years & $63.0 \pm 8.0$ & $63.0 \pm 8.5$ & -0.070 & 0.252 \\
Gender, female/ male & $13 / 10$ & $10 / 15$ & 1.310 & 0.659 \\
Education, years & $9.261 \pm 4.474$ & $9.800 \pm 3.937$ & -0.444 & 0.385 \\
Age range, years & $50-77$ & $37-74$ & & 0.077 \\
MMSE score & $29.043 \pm 1.022$ & $28.760 \pm 1.200$ & 1.809 & 0.744 \\
MoCA score & $27.130 \pm 1.180$ & $26.560 \pm 1.003$ & 0.329 & 0.064 \\
PHQ-9 score & $2.565 \pm 1.121$ & $2.440 \pm 1.474$ & 0.719 & \\
GAD-7 score & $1.826 \pm 1.466$ & $1.800 \pm 1.354$ & & \\
\hline
\end{tabular}

Note: Data were expressed as mean \pm SD.

stimuli) also decreased significantly $(\mathrm{F}(1,46)=10.691, \mathrm{P}=$ 0.002 , partial $\eta^{2}=0.189$ ). However, for the amplitude elicited by the standard stimulus level, there was no significant difference between the two groups $(\mathrm{F}(1,46)=2.115, \mathrm{P}=0.153$, partial $\left.\eta^{2}=0.044\right)$. In addition, we compared the amplitude of $\mathrm{P} 3 \mathrm{~b}$ and $\mathrm{P} 3 \mathrm{a}$ at each electrode between the two groups, and all of the above results were statistically significant $(\mathrm{P}<0.05)$. The amplitude of $\mathrm{P} 3$ showed a remarkable main effect of site $(\mathrm{F}$ $(1.346,61.934)=9.438, P=0.001$, partial $\left.\eta^{2}=0.170\right)$ (Figure 2).

In terms of P3 latency, aCSVD patients displayed a longer P3 latency $(428.564 \pm 49.700 \mathrm{~ms})$ than healthy controls $(410.604 \pm 45.591 \mathrm{~ms}, \mathrm{~F}(1,46)=10.176, \mathrm{P}=$ 0.003 , partial $\left.\eta^{2}=0.181\right)$, and a remarkable stimulus effect $\left(\mathrm{F}(2,92)=26.422, \mathrm{P}=0.000\right.$, partial $\left.\eta^{2}=0.181\right)$ was obtained. For the stimulus effect, post hoc comparisons showed that $\mathrm{P} 3 \mathrm{~b}$ (elicited by target stimuli) had the longest duration (438.393 $\pm 32.671 \mathrm{~ms})$. There was a remarkable group $\times$ stimulus interaction $(\mathrm{F}(2,92)=5.416, \mathrm{P}=0.006$, partial $\eta^{2}=0.105$ ) (Table 2). Further post hoc analysis also indicated that P3a and P3b latency of the aCSVD group was significantly longer than that of the healthy controls ( $F$ $(1,46)=21.029, \mathrm{P}<0.001$, partial $\eta^{2}=0.314$ for $\mathrm{P} 3 \mathrm{a} ; \mathrm{F}$ $(1,46)=5.473, \mathrm{P}=0.024$, partial $\eta^{2}=0.106$ for $\left.\mathrm{P} 3 b\right)$. There was no significant difference in the latency elicited by the standard stimulus level between the patients and controls $\left(\mathrm{F}(1,46)=0.001, \mathrm{P}=0.979\right.$, partial $\left.\eta^{2}=0.000\right)$ (Figure 3).

\section{NI Component}

As shown in Table 2, Figures 2 and 3, there were no statistically significant differences between the two groups for N1 amplitude and latency $(\mathrm{P}>0.05)$.

\section{P2 Component}

For P2 amplitude, we found no significant difference between the two groups $(\mathrm{F}(1,46)=1.645, \mathrm{P}=0.206$, partial $\left.\eta^{2}=0.035\right)$ or interactions of group $\times$ stimulus $(F$ $(1.397,64.281)=1.391, \mathrm{P}=0.252$, partial $\left.\eta^{2}=0.029\right)$. For P2 latency, although there was no difference between the groups $\left(F(1,46)=0.933, P=0.339\right.$, partial $\left.\eta^{2}=0.020\right)$, we found a significant interaction of group $\times$ stimulus $(F$ $(2,92)=3.757, \quad P=0.027$, partial $\left.\eta^{2}=0.076\right)$. Surprisingly, post hoc analysis showed that there was no statistically significant difference in latency between the two groups at three different stimulus levels; see Figures 2 and 3 , Table 2 .

\section{N2 Component}

There were neither significant main effects of group $(\mathrm{F}(1$, 46) $=0.001, \mathrm{P}=0.971$, partial $\eta^{2}=0.000$ ) nor remarkable interactions of group $\times$ stimulus $(F(1.777,81.735)=$ $0.252, \mathrm{P}=0.752$, partial $\left.\eta^{2}=0.005\right)$ for amplitude. $\mathrm{N} 2$ latency did not differ between groups $(\mathrm{F}(1,46)=0.123, \mathrm{P}$ $=0.727$, partial $\left.\eta^{2}=0.003\right)$, but a noticeable interaction of group $\times$ stimulus $\left(F(2,92)=4.680, P=0.012\right.$, partial $\eta^{2}=$ 0.092) was found. However, for the latency elicited by three stimulus levels, post hoc analysis showed that there was no significant difference between the two groups; see Figures 2 and 3, Table 2.

\section{Characterization of Differences in ERP Data}

We further analyzed the differences in P3 between the groups and plotted the waveform, as shown in Figure 5. Moreover, Figure 6 depicts the topographical voltage distribution of the different ERPs. 


\section{Healthy controls}
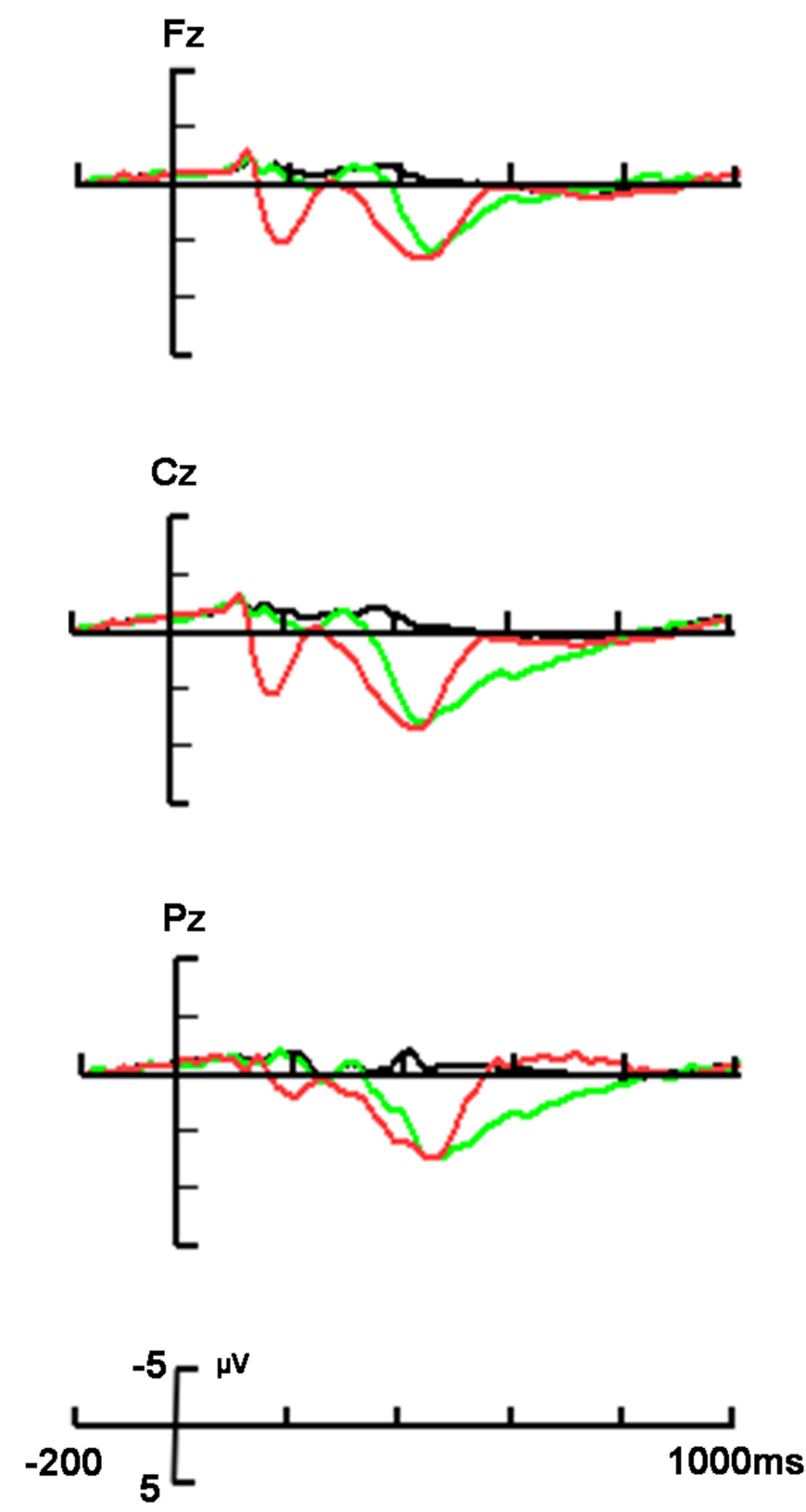

\section{aCSVD patients}
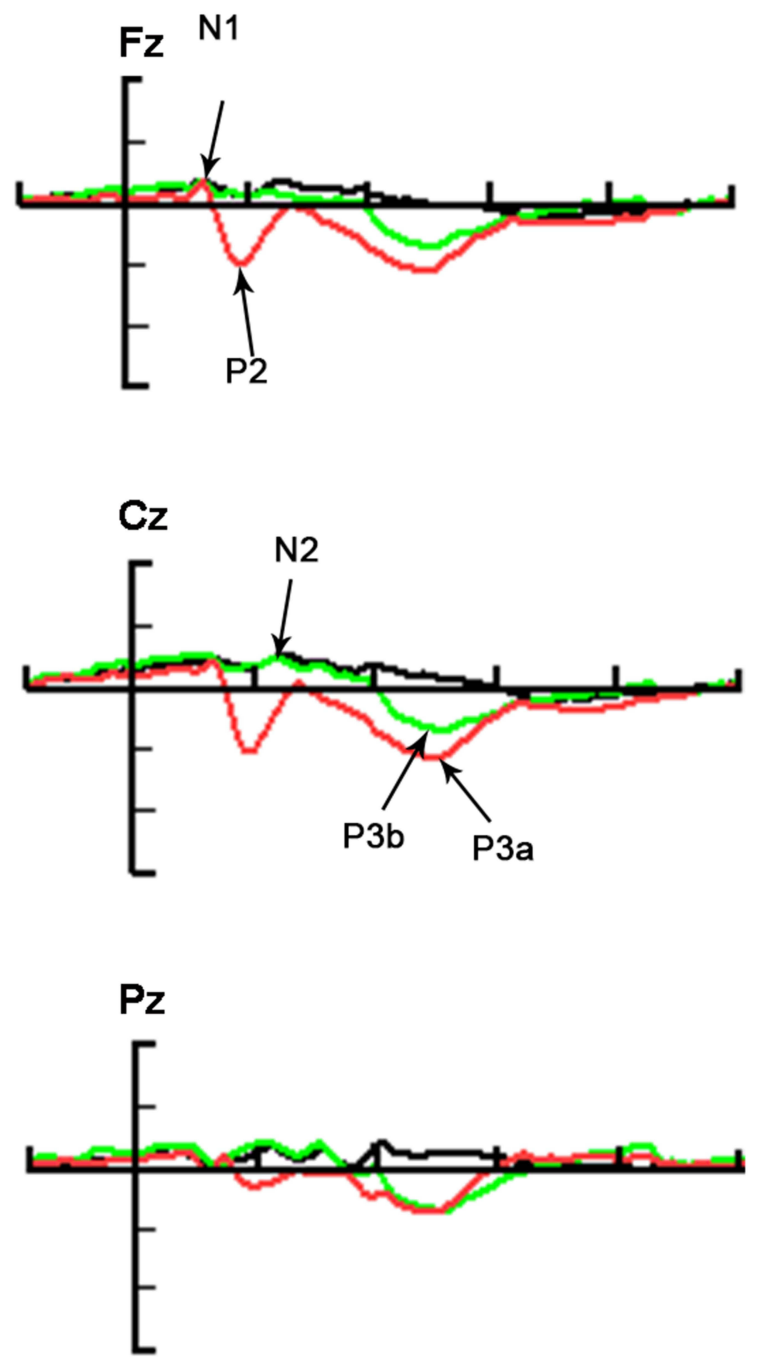

Standard stimuli

Target stimuli

\section{Novel stimuli}

Figure I The grand average waveforms of ERP in aCSVD patients and healthy controls were analyzed and drawn. Three electrodes include: Fz, Cz and Pz. The original waveforms induced by three stimuli are represented by lines of different colors: Standard (black), target (green) and novel (red line).

\section{$\mathrm{P} 3 \mathrm{~d}_{\mathrm{T}}$ and $\mathrm{P} 3 \mathrm{~d}_{\mathrm{N}}$ Components}

To observe the effects of target and novel stimuli more intuitively, we measured the $\mathrm{P} 3 \mathrm{~d}_{\mathrm{T}}$ (target minus standard) and $\mathrm{P} 3 \mathrm{~d}_{\mathrm{N}}$ (novel minus standard) components. The amplitude analysis revealed significant electrode effects in both groups $\left(\mathrm{F}(1.527,70.221)=21.061, \mathrm{P}<0.001\right.$, partial $\eta^{2}=$ 0.314 for $\mathrm{P} 3 \mathrm{~d}_{\mathrm{T}}$; $\mathrm{F}(1.575,72.470)=12.691, \mathrm{P}<0.001$, partial $\eta^{2}=0.216$ for $\left.\mathrm{P} 3 \mathrm{~d}_{\mathrm{N}}\right)$. The amplitudes were smaller for aCSVD patients $\left({\mathrm{P} 3 d_{\mathrm{T}}}_{\mathrm{T}}: 6.751 \pm 4.520 \mu \mathrm{V}, \mathrm{P} 3 \mathrm{~d}_{\mathrm{N}}: 7.620\right.$ $\pm 3.890 \mu \mathrm{V})$ than for the healthy controls $\left(\mathrm{P}^{2} \mathrm{~d}_{\mathrm{T}}: 11.071 \pm\right.$ $4.522 \mu \mathrm{V}, \mathrm{P} 3 \mathrm{~d}_{\mathrm{N}}: 11.733 \pm 3.889 \mu \mathrm{V} ; \mathrm{F}(1,46)=10.940, \mathrm{P}=$ 0.002, partial $\eta^{2}=0.192$ for $\mathrm{P} 3 \mathrm{~d}_{\mathrm{T}} ; \mathrm{F}(1,46)=13.388, \mathrm{P}$ $=0.001$, partial $\eta^{2}=0.225$ for $\mathrm{P} 3 \mathrm{~d}_{\mathrm{N}}$ ), and the significant interaction of group $\times$ electrode $(\mathrm{F}(1.527,70.221)=3.716$, 


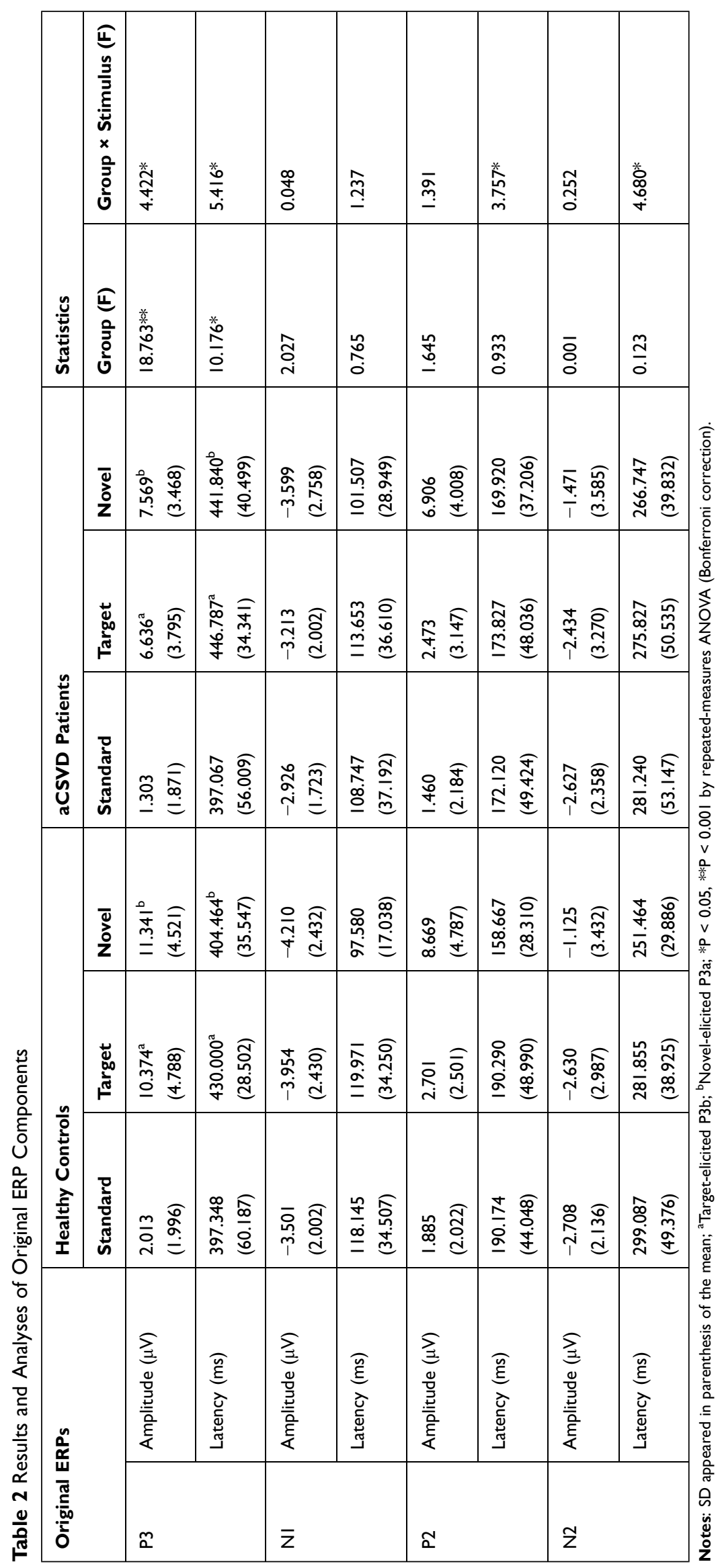




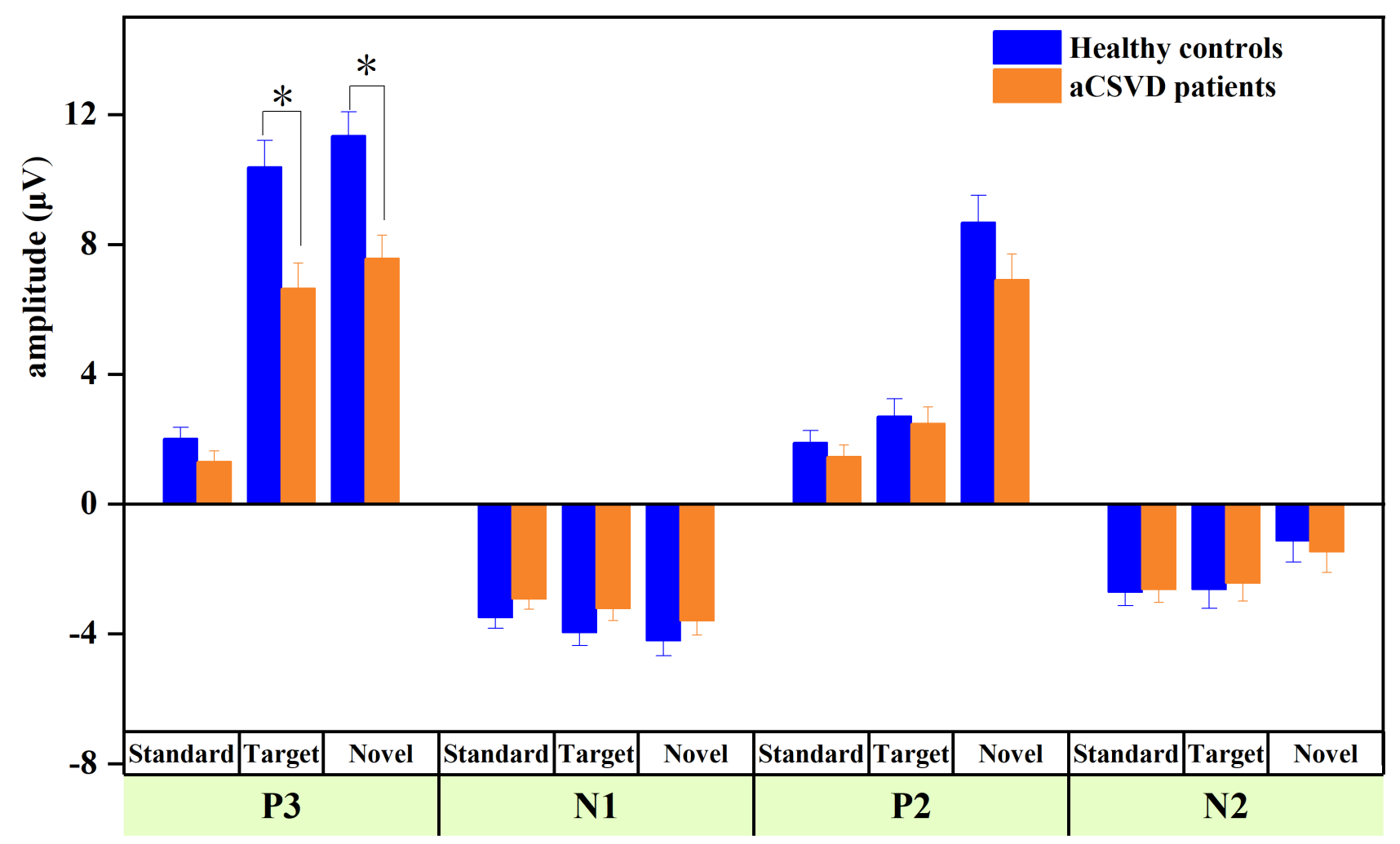

Figure 2 The amplitude of original ERP components for different expressions in two groups, $* \mathrm{P}<0.05$.

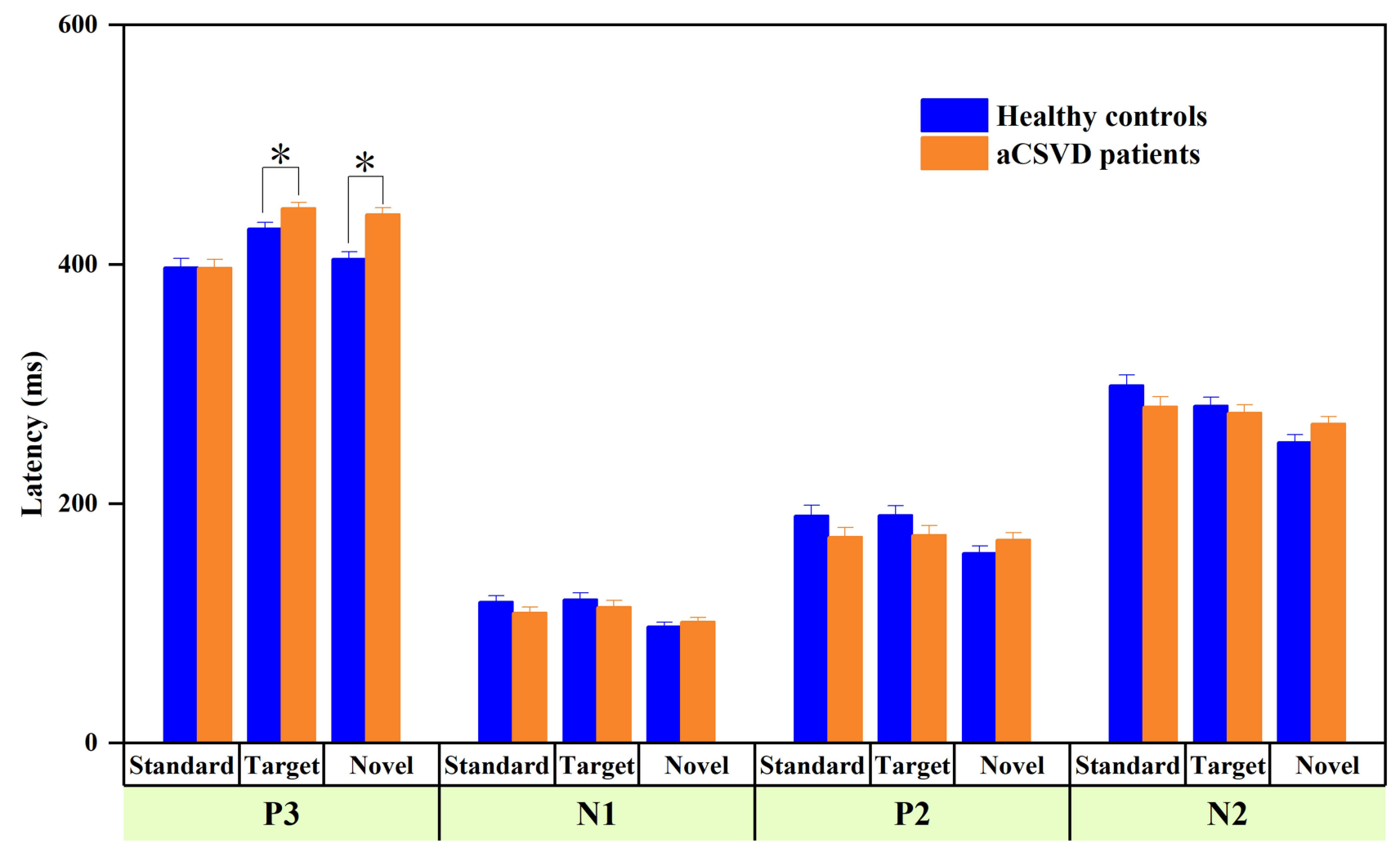

Figure 3 The latency of original ERP components for different expressions in two groups, *P $<0.05$. 


\section{A Standard stimuli B Target stimuli C Novel stimuli}
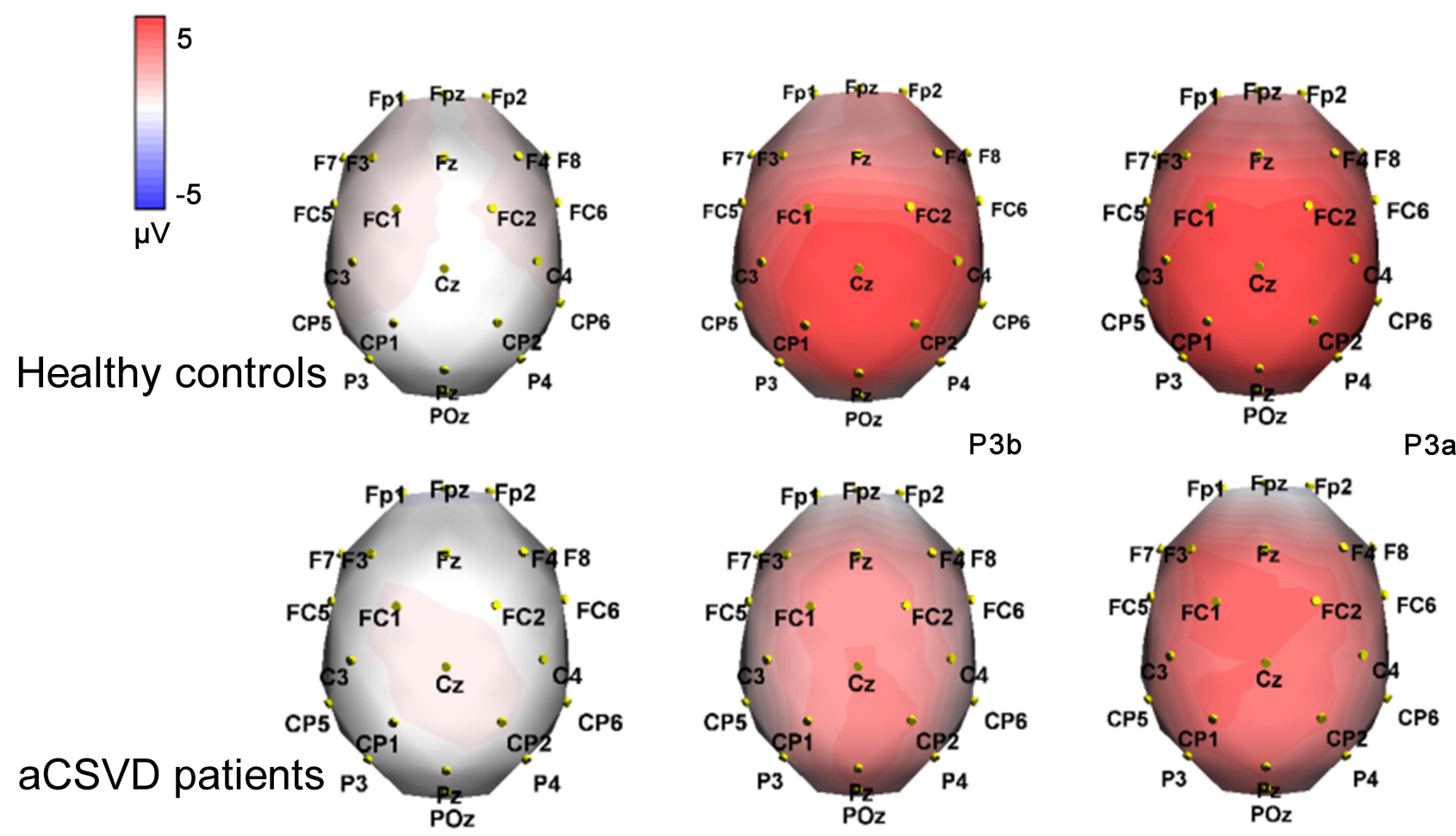

Figure 4 The above figure shows the topographical voltage distribution of P3 in aCSVD patients and healthy controls (red indicates positive potential and blue indicates negative potential). (A) standard stimulus was $400-430 \mathrm{~ms}$; (B) target stimulus was $430-450 \mathrm{~ms}$; (C) new stimulus was in $400-440 \mathrm{~ms}$.

$\mathrm{P}=0.040$, partial $\left.\eta^{2}=0.075\right)$ was observed for $\mathrm{P} 3 \mathrm{~d}_{\mathrm{T}}$ but not for $\mathrm{P} 3 \mathrm{~d}_{\mathrm{N}}(\mathrm{F}(1.575,72.470)=2.048, \mathrm{P}=0.146$, partial $\eta^{2}=0.043$ ) (Table 3 ). Subsequent comparisons showed that patients exhibited decreased $\mathrm{P} 3 \mathrm{~d}_{\mathrm{T}}$ amplitudes at all electrodes $(\mathrm{P}<0.05)$ (Figure 7$)$.

$\mathrm{P} 3 \mathrm{~d}_{\mathrm{T}}$ and $\mathrm{P} 3 \mathrm{~d}_{\mathrm{N}}$ latencies were longer in aCSVD patients $\left(\mathrm{F}(1,46)=5.483, \mathrm{P}=0.024\right.$, partial $\eta^{2}=0.107$ for $\mathrm{P} 3 \mathrm{~d}_{\mathrm{T}} ; \mathrm{F}(1,46)=22.430, \mathrm{P}<0.001$, partial $\eta^{2}=0.328$ for $P 3 d_{N}$ ) than in the healthy controls, while the remaining effects or interactions were not marked (Table 3, Figure 8).

\section{Correlations Between Attentional ERP Components and Clinical Characteristics}

We examined the amplitude and latency of $\mathrm{P} 3, \mathrm{P} 3 \mathrm{~d}_{\mathrm{T}}$, and $\mathrm{P} 3 \mathrm{~d}_{\mathrm{N}}$ induced by the three types of stimuli applied to explore in depth the relationship between ERP indexes and the clinical characteristics of aCSVD patients. Our findings revealed that the amplitude and latency of P3a showed significant and moderate correlations with Fazekas scores. In addition, the P3b amplitude was moderately correlated with the Fazekas grade, while the remaining
ERP indexes did not correlate with aCSVD characteristics (Table 4).

\section{Discussion}

Silent cerebral small vessel disease is a highly prevalent cerebrovascular pathology that usually progresses slowly with an insidious onset and is a major cause of diffuse white matter lesions and vascular cognitive impairment. ${ }^{31}$ In our research, which involved a three-stimulus visual oddball paradigm, compared with the healthy controls, aCSVD patients exhibited lower amplitude and prolonged latency of $\mathrm{P} 3, \mathrm{p} 3 \mathrm{~d}_{\mathrm{T}}$, and $\mathrm{p} 3 \mathrm{~d}_{\mathrm{N}}$. Moreover, the degree of cerebral white matter lesions was moderately correlated with these cognitive ERP abnormalities in patients. All of the results described above strongly suggest that aCSVD patients have abnormal visuospatial attention, such as orientation, target processing, and information processing speed, especially when faced with uncommon stimuli, which may be regulated by some clinical variables. The regulatory effect of visual attention on brain visual cognition is mainly reflected in the change of neural activity in the primary visual cortex (V1), ${ }^{32}$ 


\section{A Target minus Standard}
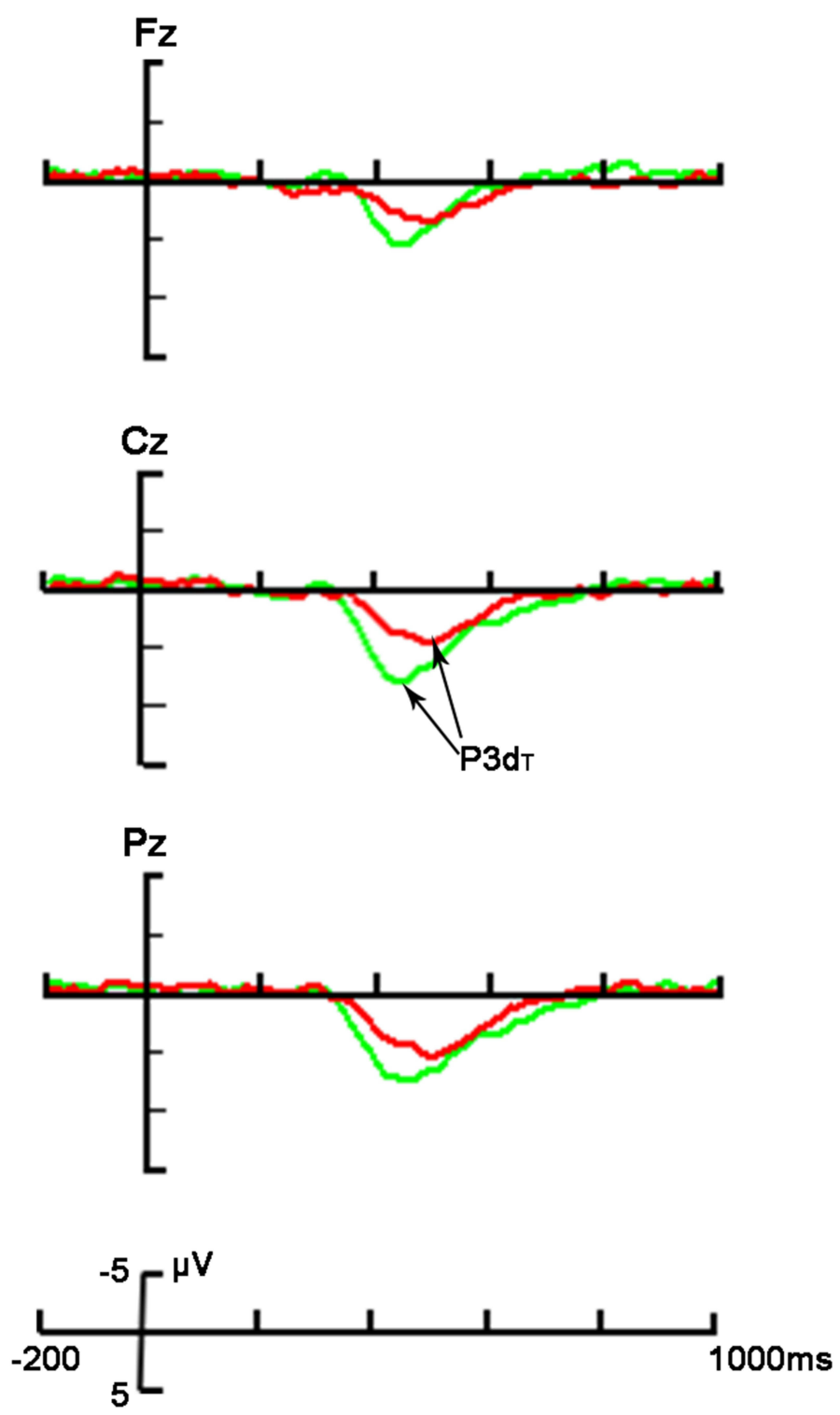

B Novel minus Standard
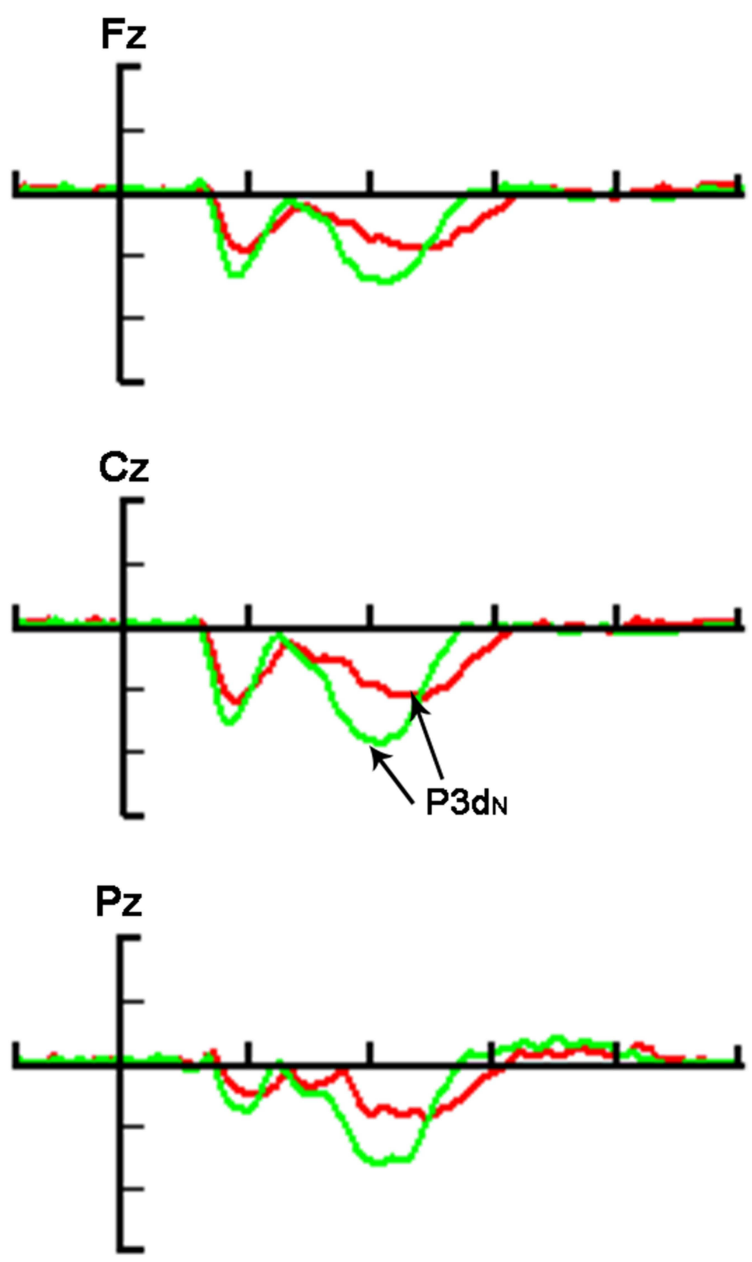

Healthy controls aCSVD patients

Figure 5 The above description and analysis were the averaged difference waveforms between the two groups (red line represents aCSVD patients and green line represents healthy controls). The electrode position: Fz, Cz, Pz. (A) Target minus standard difference ERPs (P3d $\mathrm{d}_{\mathrm{T}}$ ); (B) novel minus standard difference ERPs (P3d $\mathrm{d}_{\mathrm{N}}$ ).

which is affected by two factors: top-down (goal driven) and bottom-up (interference driven). The top-down signals are mainly transmitted from the prefrontal lobe to the frontal eye movement area and the posterior parietal cortex; the bottomup signals are transmitted from the visual cortex and occipital nucleus to the posterior parietal cortex. Studies ${ }^{33}$ have also shown that lateral posterior nucleus activity improves fundamental visual processing functions through a feedforward, surround suppression mechanism mediated by L1 inhibitory neurons. The abnormal visuospatial attention in aCSVD patients may result from diminished integration of topdown modulation or destabilized local ensembles in V1.

Basically, P3 is a positive wave usually occurring almost $300 \mathrm{~ms}$ after stimulus onset. This component reflects automatic selection of input information, which is closely related to focused attention, discrimination and execution. ${ }^{34}$ The amplitude of $\mathrm{P} 3$ might reflect the capability of information processing by the central nervous system, while its latency represents the speed of perceiving and processing stimuli. ${ }^{35} \mathrm{P} 3$ is usually subdivided into 


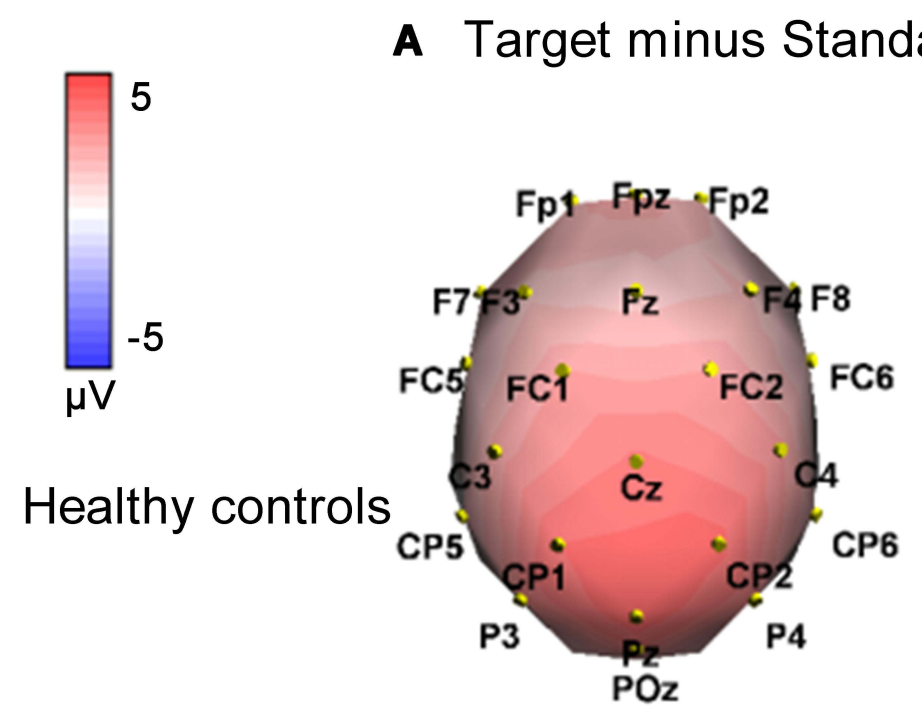

\section{B Novel minus Standard}
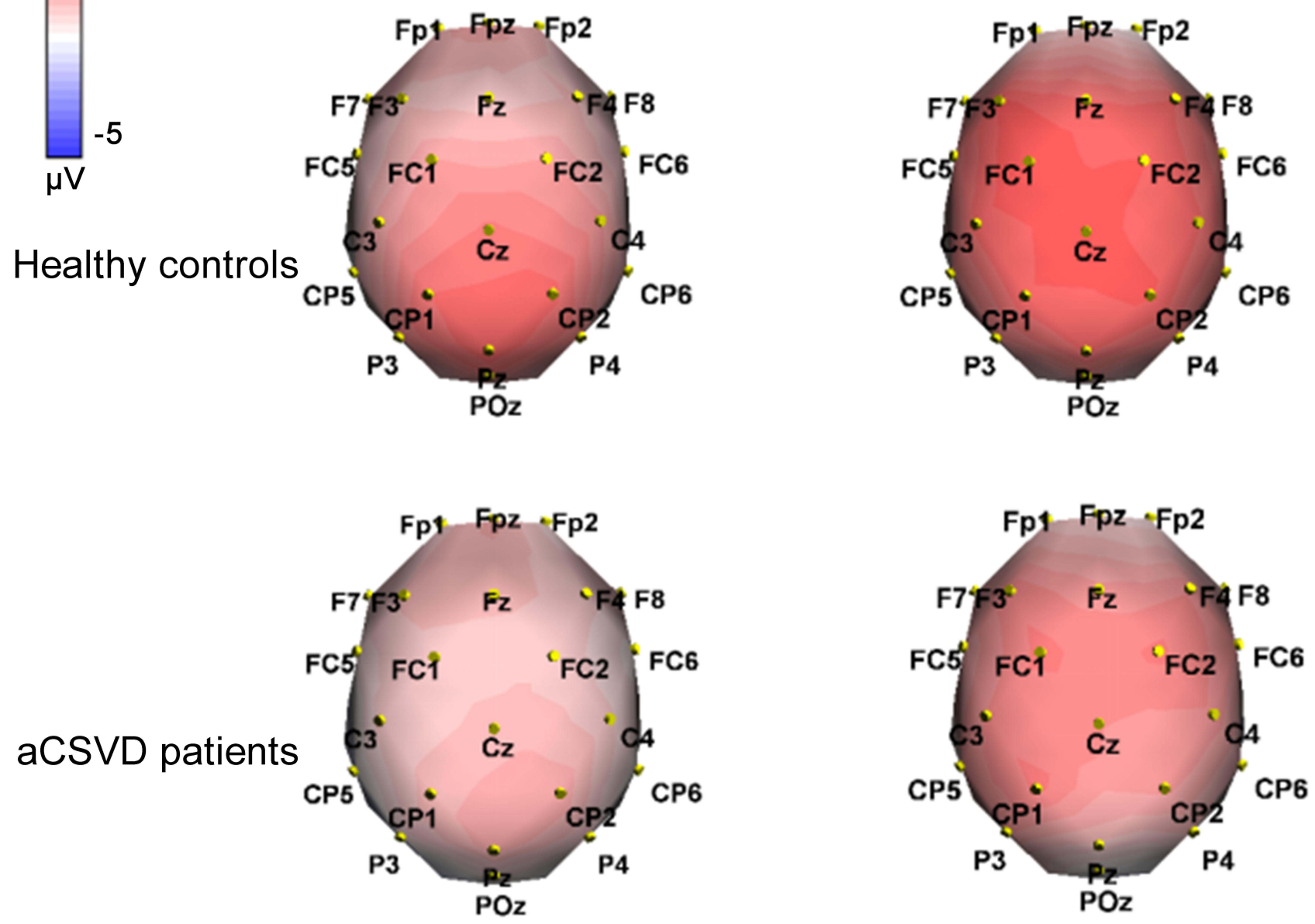

Figure 6 Analyzing respectively the topographical voltage distribution of the two groups (red indicates positive potential, blue represents negative potential). P3d (target minus standard) and P3d $\mathrm{d}_{\mathrm{N}}$ (novel minus standard) represent P3 target and novel effects. (A) P3d at $420-450$ ms; (B) P3d $\mathrm{d}_{\mathrm{N}}$ at $380-440 \mathrm{~ms}$.

$\mathrm{P} 3 \mathrm{a}$ and P3b. ${ }^{36}$ In our study, P3 elicited by the target was defined as $\mathrm{P} 3 \mathrm{~b}$, and that elicited by novel stimuli was defined as P3a. Moreover, P3a, referring to early P3, the peak latency in the visual paradigm, is $300-450 \mathrm{~ms}$, which is considered to be part of the directional response. ${ }^{37} \mathrm{P} 3 \mathrm{a}$ reflects involuntary attention and is related to the relatively automatic processing of unexpected stimuli. ${ }^{38}$ The component may be viewed as an electrophysiological manifestation of distractibility or involuntary attention shifts, since P3a is typically elicited in discrimination paradigms by stimuli extraneous to the task. ${ }^{39}$ In contrast, P3b, also known as late P3, has a peak latency of approximately $350-450 \mathrm{~ms}$, reflecting target information processing, because it is usually caused by task-related stimuli that participants actively focus on. ${ }^{40}$ This component represents the decision-making stage of cognitive processing and reflects the active awareness of target stimuli. ${ }^{41}$

In the present study, there were significant group effects and group $\times$ stimulus interactions in the amplitude and latency of P3. Post hoc analysis showed that the amplitude of P3a (novel induced) decreased and the latency was significantly prolonged. This indicates that the patient's ability to capture the unexpected stimulus or direction is decreased, and the reaction time is prolonged. When an event is deviant, a greater proportion of attentional resources needs to be directed toward it to process and categorize. This kind of orientation response can make the body aware of and deal with unexpected events prior to cognitive processing, which has important protective significance for the body. Our results imply that aCSVD 


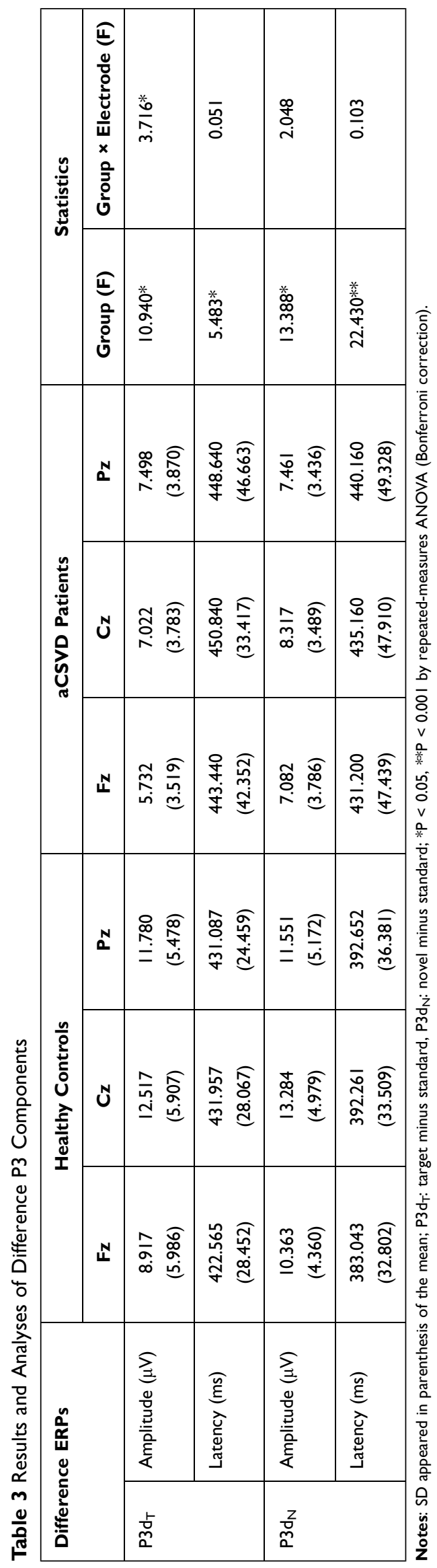

patients have deficits in preattentive visual processing of novel information. Compared with the control group, the amplitude of P3b in aCSVD patients was decreased, and the latency of P3b also increased significantly. P3b amplitude is known to reflect the information processing capacity, and these results imply that the aCSVD groups have defects in allocating attentional resources for target task information processing. $\mathrm{P} 3$ components (P3a combined with $\mathrm{P} 3 \mathrm{~b}$ ) would help provide more extensive information on the visual processing deficits of patients with aCSVD. The above findings were also confirmed in $\mathrm{P} 3 \mathrm{~d}_{\mathrm{T}}$ and $\mathrm{P} 3 \mathrm{~d}_{\mathrm{N}}$ components. A previous study demonstrated that P3 latency was prolonged, while there was no significant difference in P3 amplitude between cerebral small vessel disease patients both with and without cognitive impairment. ${ }^{42}$ The abovementioned findings were not entirely consistent with our own, and this divergence may be due to different experimental procedures or to the diversity in sample selection and grouping.

$\mathrm{N} 1$ is a preemptive negative potential involved in perception and is distributed predominantly over the frontocentral region of the scalp. ${ }^{43}$ Contrary to our expectations, we found no $\mathrm{N} 1$ abnormalities in aCSVD patients. N2 negativity is related to selective stimulus discrimination, ${ }^{44}$ target selection, and task supervision, and the maximum amplitude elicited by visual stimulation mainly exists in the occipitoparietal region. For the N2 component, there was no significant difference between the two groups at the three stimulation levels. The aforementioned results implied that the upper stream of the processing procedure (N1 and N2) remained normal for aCSVD patients, at least as suggested in our study.

P2 is sensitive to specific stimuli and is related to voluntary attention or the stimulus-encoding process. It is called an autogenerated exogenous response whose amplitude and latency reflect selective attention. This component is generally regarded as an exogenous response and is produced automatically, with its amplitude and latency reflecting selective attention. ${ }^{45}$ In our study, although the amplitude was not statistically significant, the group $x$ stimulus interaction was remarkable in P2 latency. However, this interesting finding and potential mechanism need to be verified in a larger cohort.

To clarify the relationship between ERP components (attention-related) and clinical characteristics (cognitive scale scores and imaging features) of individuals with aCSVD, Pearson's correlations were calculated. We discovered that the amplitudes of $\mathrm{P} 3 \mathrm{a}$ and $\mathrm{P} 3 \mathrm{~b}$ were 


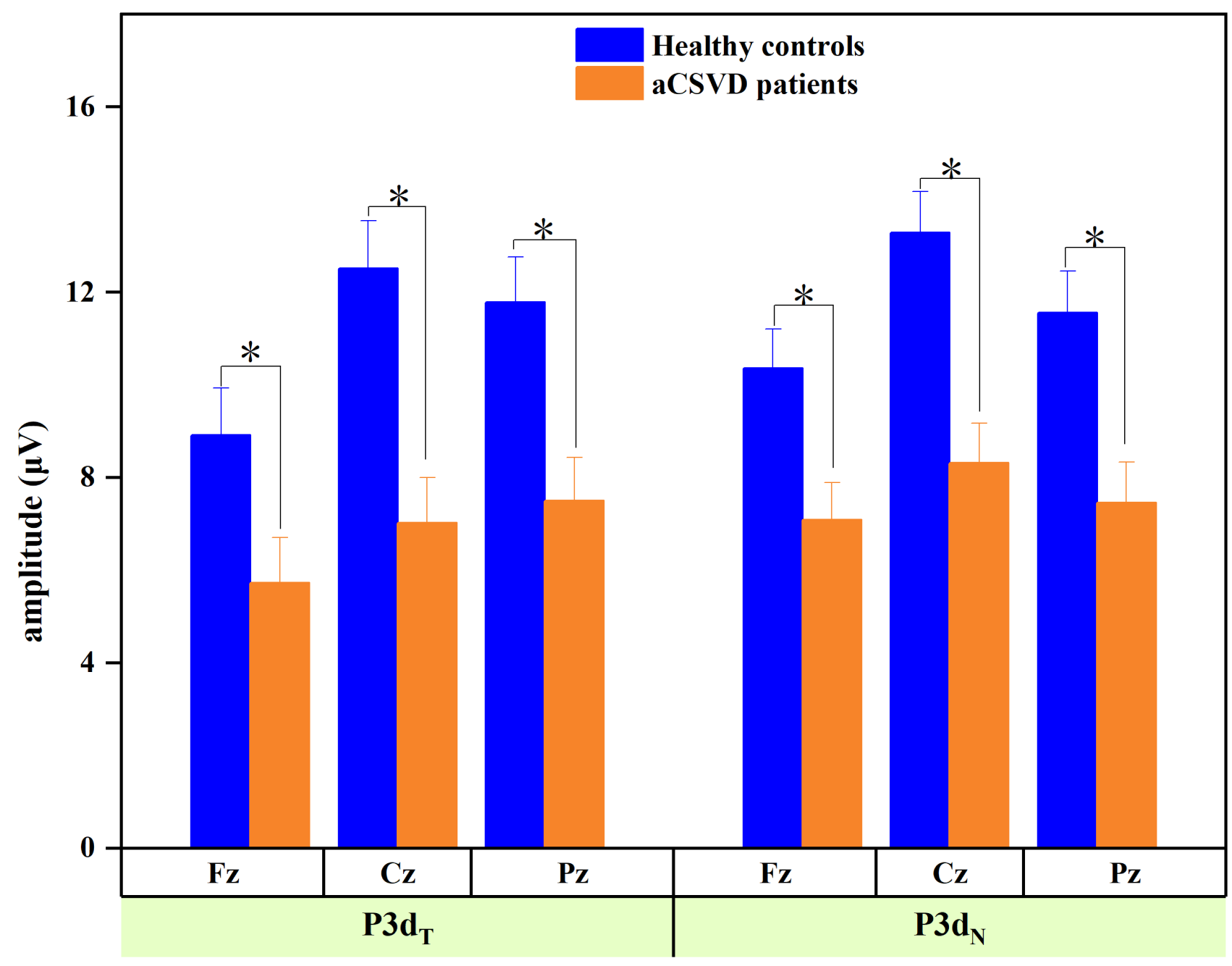

Figure 7 The amplitude of difference ERP components for different expressions in two groups, $* P<0.05$.

negatively correlated with the Fazekas score, while P3a latency was positively correlated with the Fazekas score, suggesting that the more severe the white matter lesions were, the more severe the brain dysfunction and neurocognitive impairment, especially in visual spatial attention. Cognitive ERPs had no correlation with scales (MMSE, MoCA, GAD-7, PHQ-9). Similarly, Papaliagkas et al found that MMSE and MoCA scores had no correlation with P3 amplitude and latency in CSVD patients. It was assumed that, in this study, the degree of cognitive impairment in aCSVD patients was mild and did not reach the level of severe dementia; however, asymptomatic patients with small vessel disease still had cognitive processing impairment, and the severity was potentially affected by some imaging parameters.

In summary, our study indicated that although the cognitive impairment of aCSVD patients was mild and neuropsychological tests were still in the normal range, the brain exhibited slow processing speed and poor visual spatial attention. It also implied that the ability of aCSVD patients to distinguish stimuli, process target stimuli, and deal with novel information was impaired. In particular, the ability to evaluate and respond to emergencies decreased. In addition, P3 was significantly correlated with white matter lesions, which provides more evidence for the grading of cognitive impairment. On balance, P3 components are more sensitive to the visual processing deficits of patients with aCSVD.

To the best of our knowledge, this was the first study to compare ERP components associated with visual spatial attention between healthy controls and aCSVD patients whose cognitive scales were in the normal range. Standardized selection criteria and detailed information on the clinical features of subjects were some of the advantages of this research. A three-stimulus oddball paradigm with satisfying sensitivity and reliability was employed to examine attentional visual processing, and 


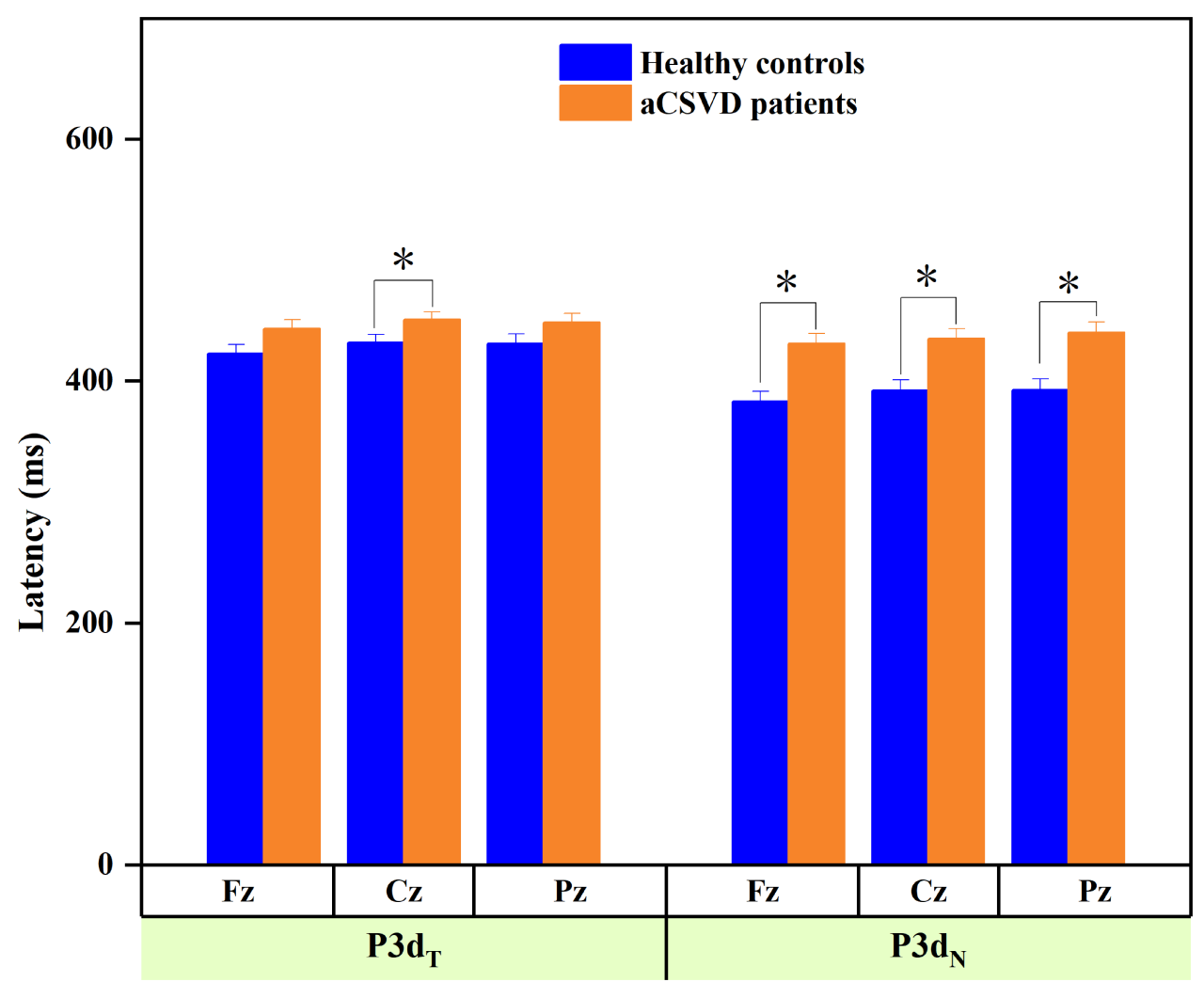

Figure 8 The latency of difference ERP components for different expressions in two groups, $* P<0.05$.

the combined analyses of voltage topographical maps and difference waveforms allowed the vivid depiction of scalp topographical distributions and eliminated the influence of the baseline effect to the minimum extent, which undoubtedly made the results more convincing. However, limitations still existed in our study. First, owing to the small cohort of subjects, we did not analyze the potential influence of age and gender. In addition, we did not discuss the differences between different imaging subtypes of cerebral small vessel disease patients. Besides, we have not taken into account the role of isolated silent lacunar infarcts versus pure cerebral white matter lesions in the sample of patients analyzed, and further studies are needed to explore the distinctions. Finally, source analysis of deviant ERP components in aCSVD patients was not performed, which we aim to explore in the near future.

Table 4 Correlations Between Attentional ERP Components and Clinical Characteristics in aCSVD Patients

\begin{tabular}{|l|l|l|l|l|l|}
\hline \multirow{2}{*}{ Original/Difference ERPs } & MMSE & MoCA & Fazekas & PHQ-9 & GAD-7 \\
\cline { 2 - 6 } & $\mathbf{r}$ (p value) & $\mathbf{r}$ (p value) & $\mathbf{r}$ (p value) & $\mathbf{r}$ (p value) & $\mathbf{r}(\mathbf{p}$ value) \\
\hline P3 amplitude-standard & $-0.225(0.052)$ & $-0.115(0.328)$ & $-0.154(0.186)$ & $0.178(0.126)$ & $-0.214(0.065)$ \\
P3 amplitude-target (p3b) & $-0.054(0.643)$ & $-0.059(0.614)$ & $-0.321(0.005)^{*}$ & $-0.071(0.544)$ & $-0.151(0.197)$ \\
P3 amplitude-novel (p3a) & $0.034(0.771)$ & $0.125(0.284)$ & $-0.377(0.001)^{*}$ & $0.011(0.927)$ & $0.069(0.556)$ \\
P3 latency-standard & $-0.099(0.398)$ & $-0.078(0.505)$ & $0.107(0.363)$ & $-0.042(0.723)$ & $-0.132(0.259)$ \\
P3 latency-target (p3b) & $0.042(0.719)$ & $-0.025(0.828)$ & $-0.192(0.100)$ & $-0.042(0.719)$ & $0.107(0.361)$ \\
P3 latency-novel (p3a) & $-0.133(0.256)$ & $-0.127(0.277)$ & $0.338(0.003)^{*}$ & $-0.093(0.426)$ & $0.100(0.393)$ \\
P3d amplitude & $0.018(0.878)$ & $-0.057(0.625)$ & $0.005(0.963)$ & $-0.085(0.470)$ & $0.094(0.422)$ \\
P3d amplitude & $0.033(0.779)$ & $-0.161(0.168)$ & $-0.193(0.097)$ & $0.152(0.194)$ & $0.116(0.320)$ \\
P3d latency & $-0.195(0.093)$ & $0.063(0.589)$ & $0.152(0.193)$ & $-0.194(0.096)$ & $0.138(0.238)$ \\
P3d latency & $-0.001(0.994)$ & $0.031(0.789)$ & $0.053(0.652)$ & $-0.109(0.354)$ & $0.104(0.374)$ \\
\hline
\end{tabular}

Notes: $\mathrm{P} 3 \mathrm{~d}_{\mathrm{T}}$ : target minus standard, $\mathrm{P} 3 \mathrm{~d}_{\mathrm{N}}$ : novel minus standard; $* \mathrm{P}<0.05$. 


\section{Conclusions}

In the above studies, we demonstrated that the amplitude of $\mathrm{P} 3 / \mathrm{P} 3 \mathrm{~d}_{\mathrm{T}} / \mathrm{P} 3 \mathrm{~d}_{\mathrm{N}}$ decreased, and post hoc analysis showed that the amplitude of $\mathrm{P} 3 \mathrm{a}$ elicited by novel stimuli and $\mathrm{P} 3 \mathrm{~b}$ elicited by target stimuli was lower in individuals with aCSVD, while the latency of P3a and P3b was prolonged. All of the data above indicated that the presence of visual space is disturbed in aCSVD (especially for rare stimuli). Considering that the cognitive impairment of aCSVD patients is often characterized by attention, execution and memory are relatively reserved. P3 may become an important electrophysiological index for distinguishing individuals with aCSVD from healthy people. ERP examination might offer a useful method with high sensitivity for identifying cerebral small vessel disease, especially in the early or subclinical phase, and for detecting dynamic cognitive alterations when combined with neuropsychological assessments. However, the availability of electrophysiological diagnostic tools for cerebral small vessel disease demands further validation.

\section{Ethical Standards}

All procedures were conducted in accordance with the declaration of Helsinki.

\section{Acknowledgment}

Thank you to all participants in the project.

\section{Funding}

This work was supported by grants from the Key R \& D projects in Shandong Province (Nos. 2019GSF108101).

\section{Disclosure}

The authors have no conflicts of interest to declare.

\section{References}

1. Hachinski V. World Stroke Day 2008: "little strokes, big trouble. Stroke. 2008;39(9):2407-2420 doi:10.1161/STROKEAHA 108.531681

2. Patel B, Markus HS. Magnetic resonance imaging in cerebral small vessel disease and its use as a surrogate disease marker. Int $J$ Stroke. 2011;6(1):47-59. doi:10.1111/j.1747-49496:47-59

3. Li Q, Yang Y, Reis C, Tao T, Li W, Li X. Cerebral small vessel disease. Cell Transplant. 2018;27(12):1711-1722. doi:10.1177/ 0963689718795148

4. Ma Y, Wang J, Wang Y, Yang Y. The biphasic function of microglia in ischemic stroke. Prog Neurobiol. 2017;157:247-272. doi:10.1016/j. pneurobio.2016.01.005

5. Arena F, Bardini P, Blasi F, et al. Gadolinium presence, MRI hyperintensities, and glucose uptake in the hypoperfused rat brain after repeated administrations of gadodiamide. Neuroradiology. 2019;61 (2):163-173. doi:10.1007/s00234-018-2120-3
6. Taylor ZJ, Hui ES, Watson AN, et al. Microvascular basis for growth of small infarcts following occlusion of single penetrating arterioles in mouse cortex. J Cereb Blood Flow Metab. 2016;36(8):1357-1373. doi: $10.1177 / 0271678 X 15608388$

7. Wardlaw JM, Smith EE, Biessels GJ, Cordonnier C, Fazekas F, Frayne R. Neuroimaging standards for research into small vessel disease and its contribution to ageing and neurodegeneration. Lancet Neurol. 2013;12(8):822-838. doi:10.1016/S1474-4422(13)70124-8

8. Peng D, Chen L, Chen X. Clinical practice guideline for cognitive impairment of cerebral small vessel disease. Aging Med. 2019;2 (2):64-73. doi:10.1002/agm2.12073

9. Staekenborg SS, Van Straaten EC, Van Der Flier WM, Lane R, Barkhof F, Scheltens P. Small vessel versus large vessel vascular dementia: risk factors and MRI findings. J Neurol. 2008;255 (11):1644-1651. doi:10.1007/s00415-008-0944-1

10. Pantoni L. Cerebral small vessel disease: from pathogenesis and clinical characteristics to therapeutic challenges. Lancet Neurol. 2010;9(7):689-701. doi:10.1016/S1474-4422(10)70104-6

11. Bondi MW, Jak AJ, Delano-Wood L, Jacobson MW, Delis DC, Salmon DP. Neuropsychological contributions to the early identification of Alzheimer's disease. Neuropsychol Rev. 2008;18(1):73-90. doi:10.1007/s11065-008-9054-1

12. Saykin AJ, Wishart HA, Rabin LA, Santulli RB, Flashman LA, West JD. Older adults with cognitive complaints show brain atrophy similar to that of amnestic MCI. Neurology. 2006;67(5):834-842. doi:10.1212/01.wnl.0000234032.77541.a2

13. Amariglio RE, Becker JA, Carmasin J, et al. Subjective cognitive complaints and amyloid burden in cognitively normal older individuals. Neuropsychologia. 2012;50(12):2880-2886. doi:10.1016/j. neuropsychologia.2012.08.011

14. O'brien JT, Erkinjuntti T, Reisberg B, Roman G, Dekosky ST. Vascular cognitive impairment. Lancet Neurol. 2003;2(2):89-98. doi:10.1016/s1474-4422(03)00305-3

15. Elizabeth C. Treatment of attention deficits in neurological disorders. Curr Opin Neurol. 2006;19(6):613-618. doi:10.1097/01. wco.0000247605.57567.9a

16. Howe AS, Bani-Fatemi A, De Luca V. The clinical utility of the auditory P300 latency subcomponent event-related potential in preclinical diagnosis of patients with mild cognitive impairment and Alzheimer's disease. Brain Cogn. 2014;86:64-74. doi:10.1016/j.bandc.2014.01.015

17. Hernández MD, Armitage PA, Thrippleton MJ, Chappell F, Wardlaw JM. Rationale, design and methodology of the image analysis protocol for studies of patients with cerebral small vessel disease and mild stroke. Brain Behav. 2015;5(12):e00415. doi:10.1002/brb3.415

18. Fazekas F, Chawluk JB, Alavi A, Hurtig HI, Zimmerman RA. MR signal abnormalities at $1.5 \mathrm{~T}$ in Alzheimer's dementia and normal aging. AJR Am J Roentgenol. 1987;149(2):351-356. doi:10.2214/ajr.149.2.351

19. Folstein MF, Folstein SE, Mchugh PR. Mini-mental state". A practical method for grading the cognitive state of patients for the clinician. J Psychiatr Res. 1975;12(3):189-198. doi:10.1016/0022-3956 (75) $90026-6$

20. Nasreddine ZS, Phillips NA, Bédirian V, et al. The Montreal Cognitive Assessment, MoCA: a brief screening tool for mild cognitive impairment. J Am Geriatr Soc. 2005;53(4):695-699. doi:10.1111/j.15325415.2005.53221.x

21. Spitzer RL, Kroenke K, Williams JB, Löwe B. A brief measure for assessing generalized anxiety disorder: the GAD-7. Arch Intern Med. 2006;166(10):1092-1097. doi:10.1001/archinte.166.10.1092

22. Kroenke K, Spitzer RL, Williams JB. The PHQ-9: validity of a brief depression severity measure. J Gen Intern Med. 2001;16(9):606-613. doi:10.1046/j.1525-1497.2001.016009606.x

23. Kapeller P, Barber R, Vermeulen RJ, Adèr H, Scheltens P, Freidl W. Visual rating of age-related white matter changes on magnetic resonance imaging: scale comparison, interrater agreement, and correlations with quantitative measurements. Stroke. 2003;34(2):441-445. doi:10.1161/01.str.0000049766.26453.e9 
24. Janes F, Cifù A, Pessa ME, Domenis R, Gigli GL, Sanvilli N. ADMA as a possible marker of endothelial damage. A study in young asymptomatic patients with cerebral small vessel disease. Sci Rep. 2019;9(1):14207. doi:10.1038/s41598-019-50778-w

25. Zhu S, Wei X, Yang X, Huang Z, Chang Z, Xie F. Plasma lipoprotein-associated phospholipase A2 and superoxide dismutase are independent predicators of cognitive impairment in cerebral small vessel disease patients: diagnosis and assessment. Aging Dis. 2019;10 (4):834-846. doi:10.14336/AD.2019.0304

26. Fazekas F, Kleinert R, Offenbacher H, Schmidt R, Kleinert G, Payer F. Pathologic correlates of incidental MRI white matter signal hyperintensities. Neurology. 1993;43(9):1683-1689. doi:10.1212/ wnl.43.9.1683

27. Wahlund LO, Barkhof F, Fazekas F, et al. A new rating scale for agerelated white matter changes applicable to MRI and CT. Stroke. 2001;32(6):1318-1322. doi:10.1161/01.str.32.6.1318

28. Guo Y, Xu S, Nie S, et al. Female versus male migraine: an eventrelated potential study of visual neurocognitive processing. $J$ Headache Pain. 2019;20(1):38. doi:10.1186/s10194-019-0995-y

29. Jung TP, Makeig S, Humphries C, et al. Removing electroencephalographic artifacts by blind source separation. Psychophysiology. 2000;37(2):163-178. doi:10.1111/1469-8986.3720163

30. Huang L, Dong HJ, Wang X, Wang Y, Xiao Z. Duration and frequency of migraines affect cognitive function: evidence from neuropsychological tests and event-related potentials. $J$ Headache Pain. 2017;18(1):54. doi:10.1186/s10194-017-0758-6

31. Smallwood A, Oulhaj A, Joachim C, et al. Cerebral subcortical small vessel disease and its relation to cognition in elderly subjects: a pathological study in the Oxford Project to Investigate Memory and Ageing (OPTIMA) cohort. Neuropathol Appl Neurobiol. 2012;38 (4):337-343. doi:10.1111/j.1365-2990.2011.01221

32. Hamm JP, Shymkiv Y, Han S, Yang W, Yuste R. Cortical ensembles selective for context. Proc Natl Acad Sci U S A. 2021;118(14): e2026179118. doi:10.1073/pnas.2026179118

33. Fang Q, Chou XL, Peng B, Zhong W, Zhang LI, Tao HW. A differential circuit via retino-colliculo-pulvinar pathway enhances feature selectivity in visual cortex through surround suppression. Neuron. 2020;105(2):355-369. doi:10.1016/j.neuron.2019.10.027

34. Lebedeva IS, Akhadov TA, Petriaikin AV, Kaleda VG, Barkhatova AN, Golubev SA. Some electrophysiological and hemodynamic characteristics of auditory selective attention in norm and schizophrenia. Zh Vyssh Nerv Deiat Im I P Pavlova. 2011;61(5):573-581.
35. Gironell A, García-Sánchez C, Estévez-González A, Boltes A, Kulisevsky J. Usefulness of p300 in subjective memory complaints: a prospective study. J Clin Neurophysiol. 2005;22(4):279-284. doi:10.1097/01.wnp.0000173559.60113.ab

36. Polich J. Updating P300: an integrative theory of P3a and P3b. Clin Neurophysiol. 2007;118(10):2128-2148. doi:10.1016/j. clinph.2007.04.019

37. Escera C, Alho K, Winkler I, Nätänen R. Neural mechanisms of involuntary attention to acoustic novelty and change. $J$ Cogn Neurosci. 1998;10(5):590-604. doi:10.1162/089892998562997

38. Winterer G, Egan MF, Rädler T, et al. Event-related potentials and genetic risk for schizophrenia. Biol Psychiatry. 2001;50(6):407-417. doi:10.1016/s0006-3223(01)01072-1

39. Stige S, Fjell AM, Smith L, Lindgren M, Walhovd KB. The development of visual P3a and P3b. Dev Neuropsychol. 2007;32(1):563584. doi:10.1080/87565640701361096

40. Jonkman LM, Lansbergen M, Stauder JE. Developmental differences in behavioral and event-related brain responses associated with response preparation and inhibition in a go/nogo task. Psychophysiology. 2003;40(5):752-761. doi:10.1111/14698986.00075

41. Soltani M, Knight RT. Neural origins of the P300. Crit Rev Neurobiol. 2000;14(3-4):199-224. doi:10.1615/CritRevNeurobiol. v14.i3-4.20

42. Zhang M, Chen M, Wang Q, Yun W, Zhang Z, Yin Q. Relationship between cerebral microbleeds and cognitive function in lacunar infarct. Int $J$ Med Res. 2013;41(2):347-355. doi:10.1177/ 0300060513476448

43. Mangun GR. Neural mechanisms of visual selective attention. Psychophysiology. 1995;32(1):4-18. doi:10.1111/j.1469-8986.1995. tb03400.x

44. Bennys K, Portet F, Touchon J, Rondouin G. Diagnostic value of event-related evoked potentials N200 and P300 subcomponents in early diagnosis of Alzheimer's disease and mild cognitive impairment. J Clin Neurophysiol. 2007;24(5):405-412. doi:10.1097/ WNP.0b013e31815068d5

45. Crowley KE, Colrain IM. A review of the evidence for P2 being an independent component process: age, sleep and modality. Clin Neurophysiol. 2004;115(4):732-744. doi:10.1016/j.clinph.2003. 11.021
Neuropsychiatric Disease and Treatment

\section{Publish your work in this journal}

Neuropsychiatric Disease and Treatment is an international, peerreviewed journal of clinical therapeutics and pharmacology focusing on concise rapid reporting of clinical or pre-clinical studies on a range of neuropsychiatric and neurological disorders. This journal is indexed on PubMed Central, the 'PsycINFO' database and CAS, and is the official journal of The International Neuropsychiatric Association (INA). The manuscript management system is completely online and includes a very quick and fair peer-review system which is all easy to use. Visit http://www.dovepress.com/testimonials.php to read real quotes from published authors. 